\title{
Fascicular anatomy of human femoral nerve: Implications for neural prostheses using nerve cuff electrodes
}

\author{
Kenneth J. Gustafson, PhD; ${ }^{1-2 *}$ Gilles C. J. Pinault, MD; ${ }^{2-3}$ Jennifer J. Neville, MD; ${ }^{4}$ shaq Syed, MD; ${ }^{4}$ \\ John A. Davis Jr, MD; ${ }^{5}$ Jesse Jean-Claude, MD; ${ }^{2-3}$ Ronald J. Triolo, PhD $^{1-2,5}$ \\ ${ }^{1}$ Department of Biomedical Engineering, Case Western Reserve University, Cleveland, $\mathrm{OH} ;{ }^{2}$ Louis Stokes Cleveland \\ Department of Veterans Affairs Medical Center, Cleveland, $\mathrm{OH} ;{ }^{3}$ Department of Surgery, ${ }^{4}$ School of Medicine, and \\ ${ }^{5}$ Department of Orthopedics, Case Western Reserve University, Cleveland, $\mathrm{OH}$
}

\begin{abstract}
Clinical interventions to restore standing or stepping by using nerve cuff stimulation require a detailed knowledge of femoral nerve neuroanatomy. We harvested eight femoral nerves with all distal branches and characterized the branching patterns and diameters. The fascicular representation of each distal nerve was identified and traced proximally to create fascicle maps of the compound femoral nerve in four cadaver specimens. Distal nerves were consistently represented as individual fascicles or distinct groups of fascicles in the compound femoral nerve. Branch-free length of the compound femoral nerve was $1.50+/-0.47 \mathrm{~cm}$ (mean $+/-$ standard deviation). Compound femoral nerve cross sections were noncircular with major and minor diameters of $10.50+/-1.52 \mathrm{~mm}$ and $2.30+/-0.63 \mathrm{~mm}$, respectively. In vivo intraoperative measurements in six subjects were consistent with cadaver results. Selective stimulation of individual muscles innervated by the femoral nerve may therefore be possible with a single neural prosthesis able to selectively stimulate individual groups of fascicles.
\end{abstract}

Key words: fascical map, femoral nerve, functional electrical stimulation, nerve cuff electrode, nerve morphology, neuroanatomy, rehabilitation, spinal cord injury, standing, surgical anatomy.

\section{INTRODUCTION}

Muscles innervated by the femoral nerve are prime movers for leg extension at the knee joint and thigh flexion and critical for standing and stepping function. Therefore, effective clinical interventions to restore or improve stand- ing or walking after paralysis resulting from upper motor neuron damage secondary to spinal cord injury (SCI), or peripheral nerve damage due to trauma, require a detailed knowledge of the morphology and fascicular anatomy of the femoral nerve. However, the fascicular anatomy and specific dimensions of the human femoral nerve have not been fully described and must be quantitatively documented.

The femoral nerve originates from the second, third, and fourth lumbar spinal nerves and innervates the anterior thigh muscles, hip and knee joints, and skin on the anteromedial thigh [1]. We define the compound femoral nerve as the femoral nerve section between the inguinal ligament and the first branching point in the thigh. The three vasti muscles are important for standing function; they extend the leg at the knee joint without flexing the thigh, which would be detrimental to a stable upright posture. Vastus lateralis and vastus intermedius are the strongest of the three muscles; therefore, they are commonly targeted by neural prostheses that employ functional electrical stimulation

Abbreviations: ASIS = anterior superior iliac spine, FES = functional electrical stimulation, $\mathrm{SCI}$ = spinal cord injury, SD = standard deviation.

*Address all correspondence to Kenneth J. Gustafson, PhD; Department of Biomedical Engineering, Neural Engineering Center, Wickenden Building, Rm 114, Case Western Reserve University, Cleveland, OH 44106-7207; 216368-8626; fax: 216-368-4872. Email: kjg@case.edu

DOI:10.1682/JRRD.2008.08.0097 
(FES) for standing after SCI [2-4]. The vastus medialis muscle is important for locking the knee in terminal extension and preventing the patellar drift and lateral subluxation that could possibly be caused by the pull of the vastus lateralis. Although the biarticulate rectus femoris and sartorius muscles are critically important during the sit-to-stand transition and during stepping and walking [5-6], they have undesirable actions for standing [4,7], such as thigh flexion. The sartorius also flexes the leg at the knee joint and medially rotates the leg. The pectineus muscle generates low forces easily counteracted by other thigh muscles, and the saphenous and medial cutaneous sensory nerves are unessential to standing.

Currently available neural prostheses for standing and stepping after SCI use muscle-based, rather than nervebased, electrodes. Surgically implanted FES systems employing epimysial electrodes sutured to the muscle at the nerve entry point [8-9] and/or intramuscular electrodes inserted into the muscle belly near its innervating neural structure [10] have been successful in restoring hand grasp to individuals with midcervical tetraplegia [11-12]; exercise, standing, and transfer ability to individuals with thoracic or low cervical-level lesions [2,13-15]; and shortdistance stepping for individuals with thoracic paraplegia [5-6]. In spite of these successes, placement of a nerve cuff electrode on the compound femoral nerve or its distal branches would address several significant limitations of currently available muscle-based stimulation technologies. Epimysial and intramuscular electrodes rarely recruit all the motor units in a paralyzed muscle, especially in large lower-limb muscles with complex innervation patterns, leading to limited standing durations or insufficient stimulated joint moments to support taller and heavier implant recipients. For complete recruitment using muscle-based electrodes, multiple electrodes must be placed within the muscle belly, which is surgically time consuming and can be difficult to implement [16]. Nerve cuff electrodes can completely activate the motor neurons they encompass and produce contractions closer to the maximal capacity than muscle-based designs.

Distal nerves may be represented as distinct fascicles or groups of fascicles in proximal compound peripheral nerves. A fascicle map of a compound nerve relates each fascicle to the distal nerve branch with which it exits and, therefore, to the distal muscles or organs innervated by that distal nerve branch (Figure 1). Available techniques allow us to selectively electrically stimulate individual fascicles or areas within a compound nerve [17-19].
Therefore, if distinct fascicles or groups of fascicles represent the distal nerves of the compound femoral nerve, we may possibly control distal muscles at a proximal site using a single multicontact nerve cuff electrode. A single nerve cuff electrode able to selectively recruit multiple heads of the vasti muscles simultaneously would improve neuroprosthesis performance by extending standing time and eliminating the need for multiple implants.

Our objectives for this project were to determine (1) the branching patterns, branch-free lengths, and nerve diameters of the branches of the human femoral nerve; and (2) the fascicular representation of distal nerve branches at the level of the compound femoral nerve. We require accurate nerve anatomy representations to develop accurate computer models to evaluate and optimize potential stimulating nerve cuff electrode designs for standing and stepping after SCI [20]. Nerve morphology (diameters and branch freelengths) provides important design parameters for constructing nerve cuff electrodes to avoid mechanical trauma and maximize stimulation efficiency [21-22]. Furthermore, this knowledge is critical for defining surgical approaches to the nerve, identifying the most practical and desirable locations for nerve cuff electrodes, and developing implementation strategies for advanced implanted neural prostheses. Finally, we require the fascicular structure of the compound femoral nerve and the relationship of individual fascicles to distal nerve branches and terminal muscles to determine the electrode specifications and initial feasibility of selectively activating individual fascicles within the compound nerve with a single proximally located nerve cuff electrode. Computer models using the data from this study have demonstrated that selective stimulation of the human femoral nerve is feasible using nerve cuff electrodes [23].

\section{METHODS}

\section{Anatomy and Morphology}

We harvested and dissected eight femoral nerves and all distal branches from each leg of four female formalinfixed cadavers, ranging in age from 87 to 99 years (mean = 91). We measured the distance from the anterior superior iliac spine (ASIS) to the pubic symphysis bilaterally on each cadaver as an anatomical landmark. We located the femoral nerve through transverse incisions from the ASIS to the pubic symphysis and incisions originating from the midpoint between the ASIS and the pubic symphysis extending longitudinally to the patella. We chose the 


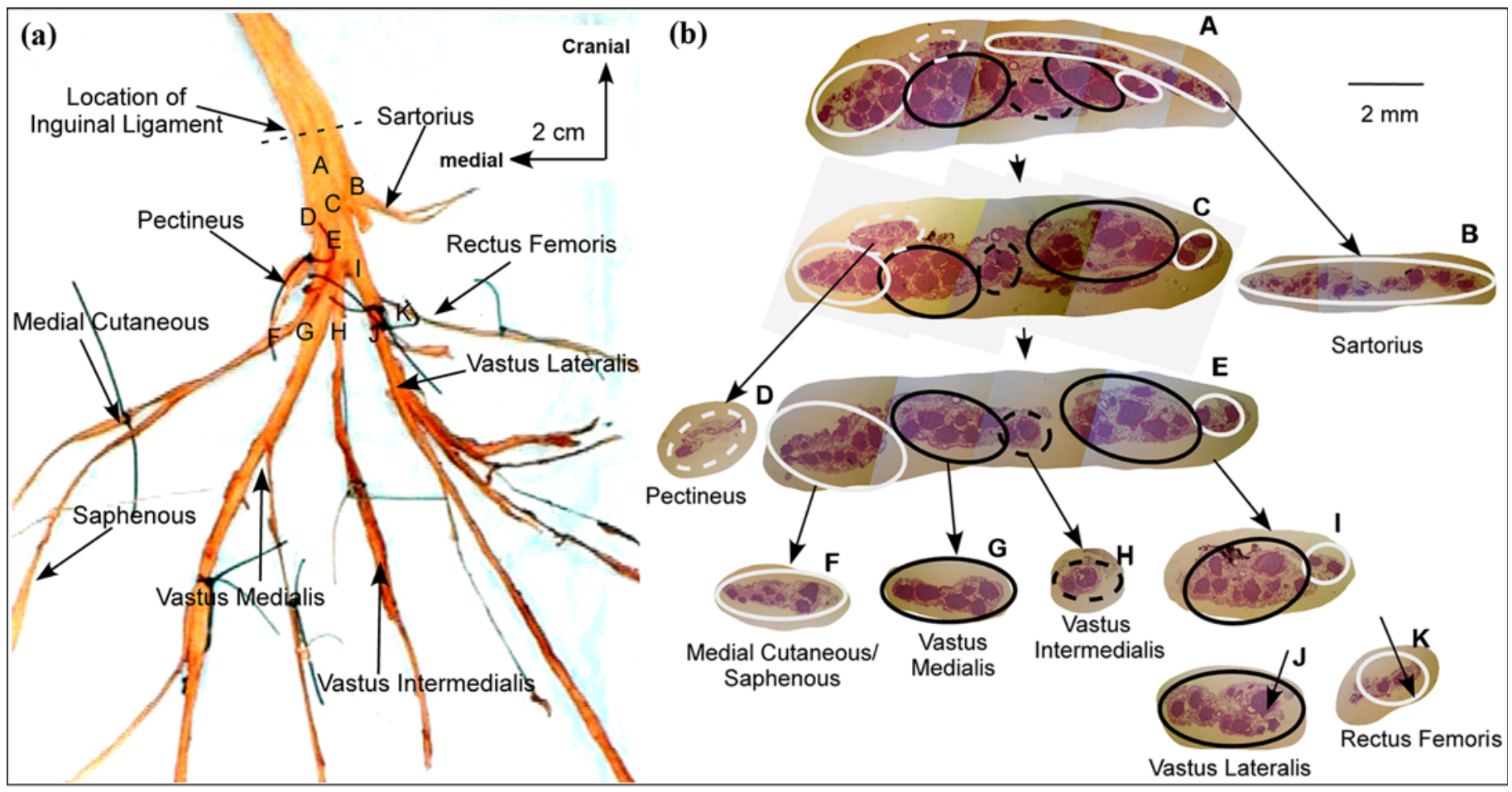

Figure 1.

(a) Harvested nerve and (b) complete fascicle map of femoral nerve (cadaver specimen 4 [left side]). We traced distal nerve branches of femoral nerve proximally to compound femoral nerve. Nerve cross sections are shown ventral side up. A = nerve just proximal to branching, $\mathrm{B}=$ nerve branch to sartorius muscle, $\mathrm{C}=$ nerve just distal to sartorius nerve branching and just proximal to pectineus nerve branching, $\mathrm{D}=$ nerve branch to pectineus muscle, $\mathrm{E}=$ nerve just distal to pectineus nerve branching and just proximal to medial cutaneous/saphenous nerve branching, $\mathrm{F}=$ nerve to medial cutaneous and saphenous nerves, $\mathrm{G}=$ nerve branch to vastus medialis muscle, $\mathrm{H}$ = nerve branch to vastus intermedius muscle, $\mathrm{I}=$ nerve to vastus lateralis and rectus femoris muscles, $\mathrm{J}=$ nerve branch to vastus lateralis muscle, $\mathrm{K}=$ nerve branch to rectus femoris muscle.

inguinal ligament as a proximal limitation for surgical dissection of the femoral nerve in the thigh.

We traced and dissected all femoral nerve branches to the insertion points. We also traced sensory branches (saphenous and medial cutaneous nerves) for completeness. We isolated nerve branches from superficial to deep to minimize damage to the nerves. As we isolated a nerve muscle entry point, we detached the muscle from its tendons and reflected it. We repeated this until we exposed all femoral nerve branches. We carefully tagged each main nerve branch with a suture to maintain orientation and identification during the dissection process.

We examined and characterized the branching patterns of all eight femoral nerves by the order of branching, both medial to lateral and proximal to distal. We measured the circumference and branch-free length of the femoral nerve and each of the branches in all eight specimens during extraction. We determined distal circumferences by measuring the length of a suture passed around the nerve just distal to branching off the com- pound femoral nerve; we tabulated branch-free lengths as the linear distances between branch points. Because cross sections were not circular, we used calipers to measure the major and minor axes of four femoral nerves just proximal to the first branching point and distal to the inguinal ligament. We measured the major and minor axes of the other four femoral nerves after histological processing. We calculated effective diameters for all nerves from the circumferences assuming a circular cross section. We took digital photographs of each nerve with its branches still attached to the muscles before we detached the nerve and extracted it. We took additional pictures and made hand drawings after we removed the nerve from the body. We stored all nerves in 10 percent buffered formalin solution for preservation.

\section{In Vivo Human Measurements}

We determined the cross-sectional dimensions of the compound femoral nerve just distal to the inguinal ligament in six subjects. All subjects were male, mean \pm standard 
deviation (SD) $62.20 \pm 5.56$ years (range 51-66). We received approval for nerve exposures in subjects undergoing elective vascular surgery from the Institutional Review Board of the Louis Stokes Cleveland Department of Veterans Affairs Medical Center. Subjects underwent standard longitudinal groin incisions to expose the common femoral artery for bypass. Concurrently, we dissected the femoral nerve at that level. We exposed the nerve and then determined the dimensions. We also measured nerve widths using surgical photographs.

\section{Fascicular Anatomy}

We sectioned four cadaver femoral nerves to trace the course of distal fascicles through the nerve to the proximal compound femoral nerve. We took $5 \mathrm{~mm}$-long transverse sections at locations just proximal and distal to all branching points and took consecutive sections proximally at $5 \mathrm{~mm}$ intervals along the femoral nerve to the inguinal ligament. We marked sections with histopathology tissue dyes to identify and verify the orientation of each section during processing. We embedded nerve samples in paraffin and took cross sections of $5 \mu \mathrm{m}$ thickness, mounted them on slides, and stained them using hematoxylin and eosin. We made digital images of each cross section through a microscope at $5 \times$ to $10 \times$ and compiled and analyzed them using Canvas 8.0 (ACD Systems; Victoria, Canada). We created complete fascicle maps from the digital images of successive sections, beginning with the terminal branches of the nerve and continuing proximally to the inguinal ligament in four cadaver specimens. We recorded the fascicle count for all sections proximal to branching, as well as for all terminal branches.

We determined the effective diameter of each individual fascicle just proximal to the first branching point in the four fully mapped specimens. We analyzed the digitized image of the nerve section using ImageJ (National Institutes of Health; Bethesda, Maryland). We manually outlined each fascicle in ImageJ and used the software to calculate the fascicular cross-sectional area. We assumed a circular approximation for all fascicles and calculated the effective diameter $\left(D_{\text {eff }}\right)$ of each fascicle as $2 \times$ $(\text { Area } / \pi)^{0.5}$.

We present data as mean \pm SD. We used the twotailed Student's $t$-test with significance criteria of 0.05 for comparisons.

\section{RESULTS}

\section{Anatomy and Morphology}

The mean medial-lateral distance from the ASIS to the pubic symphysis was $14.50 \pm 1.34 \mathrm{~cm}$ (range 13-16). We found the femoral nerve near the midpoint, $46 \pm 5$ percent (range $42 \%-52 \%$ ) from the ASIS. We did not measure either leg in cadaver specimen 3 since bony landmarks were difficult to palpate because of excessive adipose tissue. The distance from the inguinal ligament to the first branching point of the femoral nerve was $1.50 \pm 0.47 \mathrm{~cm}$ (range 1-2).

Figure 1(a) shows an example of a harvested femoral nerve. The branching pattern of the femoral nerve remained consistent between specimens. Table 1 shows the branching patterns for all specimens, including the order and medial-lateral location of each branch leaving the femoral nerve. Most branching occurred within a small length of the nerve. Branches to the pectineus muscle, sartorius muscle, and sensory nerves (saphenous and medial cutaneous) branched first and medially in seven out of eight specimens. The sartorius muscle branched laterally in one specimen. In all but one specimen, the branch to the sartorius originated on the ventral surface of the nerve. The vastus medialis, vastus intermedius, and vastus lateralis muscles were centrally located and the rectus femoris muscle was lateral in the branching pattern of all specimens.

Table 2 reports branch-free lengths and circumferences of the femoral nerve and branches. All femoral nerves had average branch-free lengths $>1.0 \mathrm{~cm}$ and effective diameters $>5.9 \mathrm{~mm}$. The femoral nerve cross sections were elongated ellipses rather than circles. Femoral nerve width was almost five times the height. Table 3 shows cross-sectional dimensions for each cadaver specimen just proximal to the first branching point. We found no statistical difference in these measurements between the left and right sides or between measurements taken pre- and posttissue processing. Figures 1(b) to 3 show examples of individual nerve cross sections.

\section{In Vivo Human Measurements}

Table 4 shows the measured width and height and calculated values for the in vivo intraoperative measurements. We found the in vivo heights to be less than those we measured in the cadavers $(p<0.005)$, resulting in a greater width/height ratio $(p<0.01)$ than those we observed in the postmortem tissue (Table 3). Although 
Table 1.

Femoral nerve branching patterns. Relative mediolateral location and order of each nerve leading femoral nerve.

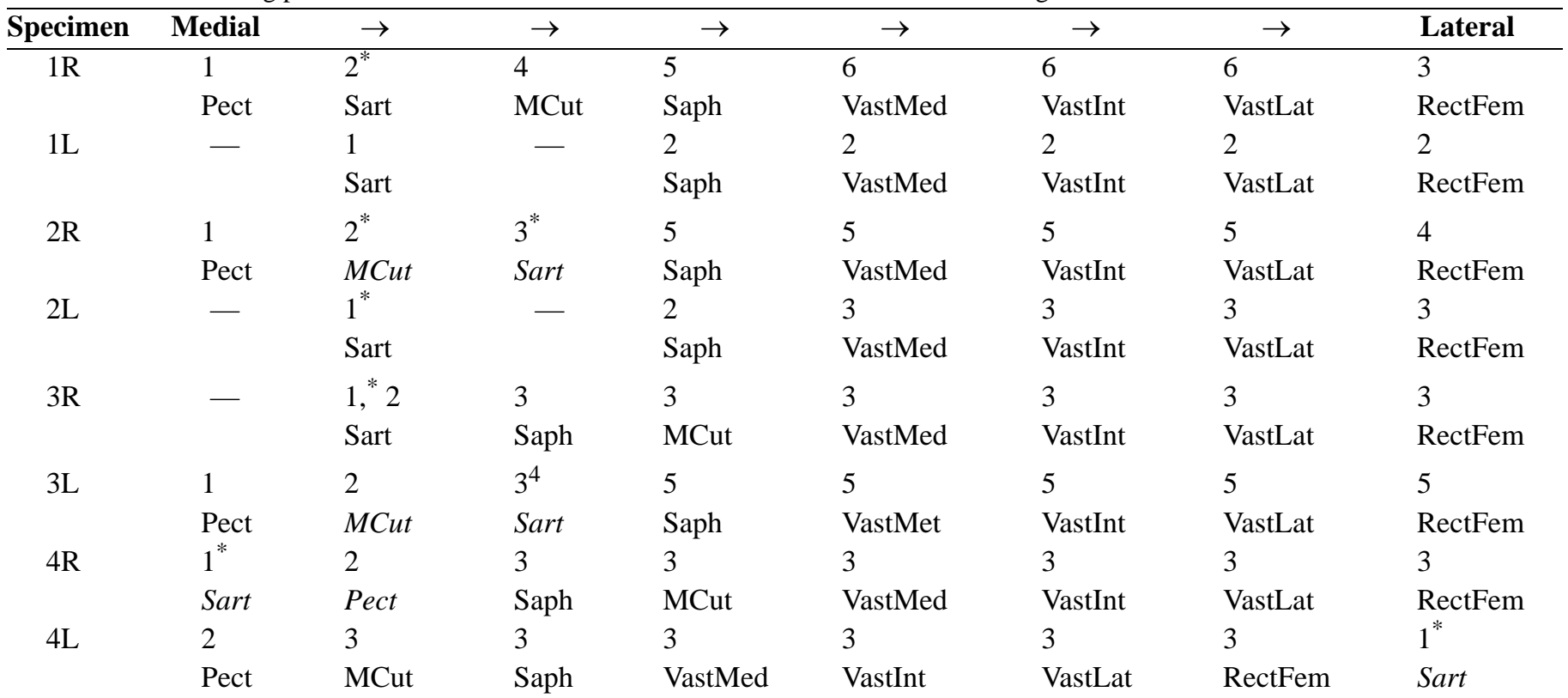

Note: Numbers denote order of branching off compound femoral nerve proximally (1) to distally (6). Italic lettering denotes deviation from normal medial to lateral branching pattern.

*Banched off ventral portion of nerve as opposed to medial or lateral sides.

— = branch not present, $\mathrm{L}=$ left side, $\mathrm{MCut}=$ medial cutaneous, Pect $=$ pectineus, $\mathrm{R}=$ right side, RectFem $=$ rectus femoris, Saph $=$ saphenous, Sart $=$ sartorius, VastInt $=$ vastus intermedius, VastLat $=$ vastus lateralis, VastMed $=$ vastus medialis.

Table 2.

Morphology of femoral nerve and all branches. Mean \pm standard deviation (range).

\begin{tabular}{lccc}
\hline \multicolumn{1}{c}{ Nerve } & Branch-Free Length (cm) & Circumference (cm) & Effective Diameter (mm) $^{*}$ \\
\hline Femoral $^{\dagger}$ & $1.50 \pm 0.47(1.0-2.0)$ & $2.50 \pm 0.67(1.8-3.9)$ & $7.9 \pm 2.1(5.9-12.0)$ \\
Vastus Medialis & $5.60 \pm 7.83(0.0-24.5)$ & $1.40 \pm 0.29(1.0-1.8)$ & $5.0 \pm 0.9(3.2-5.7)$ \\
Vastus Intermedius & $4.10 \pm 1.99(2.0-7.0)$ & $1.00 \pm 0.30(0.4-1.4)$ & $3.0 \pm 0.9(1.3-3.8)$ \\
Vastus Lateralis & $2.30 \pm 2.93(0.0-6.0)$ & $1.20 \pm 0.29(0.8-1.4)$ & $4.0 \pm 1.0(2.5-4.5)$ \\
Pectineus & $2.40 \pm 0.85(1.5-3.5)$ & $0.90 \pm 0.08(0.9-1.0)$ & $3.0 \pm 0.3(2.5-3.2)$ \\
Rectus Femoris & $2.70 \pm 3.03(0.0-9.0)$ & $1.30 \pm 0.32(1.0-1.8)$ & $4.1 \pm 1.0(3.2-5.7)$ \\
Sartorius & $6.10 \pm 4.04(1.5-12.0)$ & $1.10 \pm 0.15(0.9-1.3)$ & $3.6 \pm 0.5(2.9-4.1)$ \\
Medial Cutaneous & $19.90 \pm 7.31(10.0-28.0)$ & $1.00 \pm 0.22(0.8-1.4)$ & $3.0 \pm 0.7(2.5-4.5)$ \\
Saphenous & $21.30 \pm 11.04(4.0-35.0)$ & $1.40 \pm 0.18(1.1-1.6)$ & $4.0 \pm 0.6(3.5-5.1)$ \\
\hline
\end{tabular}

* Derived assuming circular cross section.

${ }^{\dagger}$ Midpoint between inguinal ligament and first branching point.

the in vivo widths, circumferences, and effective diameters were lower than the postmortem values, these were not significant $(p>0.05)$.

\section{Fascicular Anatomy}

Distinct fascicles or groups of fascicles in each compound femoral nerve represented distal nerve branches of the femoral nerve (sartorius, pectineus, medial cutaneous, saphenous, vastus medialis, vastus intermedius, vastus lateralis, and rectus femoris). Figure 1(b) shows an example of fascicular tracking and fascicle map construction. Fascicles maintained their spatial orientation before and after branching points. We performed successful fascicular tracing in all specimens; we found a tracing resolution (distance between longitudinal sections) of $5 \mathrm{~mm}$ to be adequate in almost all sections. 
JRRD, Volume 46, Number 7, 2009

Table 3.

Cross-sectional dimensions of compound femoral nerve from cadavers.

\begin{tabular}{cccccc}
\hline Specimen & $\begin{array}{c}\text { Width } \\
(\mathbf{m m})\end{array}$ & $\begin{array}{c}\text { Height } \\
(\mathbf{m m})\end{array}$ & $\begin{array}{c}\text { Width// } \\
\text { Height }\end{array}$ & $\begin{array}{c}\text { Circumference } \\
(\mathbf{m m})\end{array}$ & $\begin{array}{c}\text { Effective } \\
\text { Diameter } \\
(\mathbf{m m})\end{array}$ \\
\hline $1 \mathrm{R}$ & 10.5 & 2.0 & 5.3 & $18.0-19.0$ & 7.3 \\
$1 \mathrm{~L}^{\dagger}$ & 8.7 & 2.3 & 3.8 & 19.7 & 6.3 \\
$2 \mathrm{R}^{\dagger}$ & 8.5 & 1.8 & 4.7 & 18.4 & 5.9 \\
$2 \mathrm{~L}^{\dagger}$ & 10.8 & 3.5 & 3.1 & 24.3 & 7.7 \\
$3 \mathrm{R}$ & 12.7 & 1.6 & 7.9 & 39.0 & 12.4 \\
$3 \mathrm{~L}$ & 12.0 & 2.3 & 5.2 & 30.3 & 9.6 \\
$4 \mathrm{R}$ & 9.6 & 3.0 & 3.2 & 21.0 & 6.7 \\
$4 \mathrm{~L}^{\dagger}$ & 11.5 & 2.2 & 5.2 & 24.1 & 7.7 \\
\hline Mean \pm SD & $10.5 \pm 1.5$ & $2.3 \pm 0.6$ & $4.8 \pm 1.6$ & $25.0 \pm 6.7$ & $7.9 \pm 2.1$ \\
\hline${ }^{*}$ Derived assuming circular cross sections. \\
${ }^{\dagger}$ Measurements taken after tissue processing. \\
$\mathrm{L}=$ left side, R = right side, SD = standard deviation. \\
\hline \hline
\end{tabular}

Table 4.

Cross-sectional dimensions of compound femoral nerve from in vivo subjects.

\begin{tabular}{ccclcc}
\hline Subject & $\begin{array}{c}\text { Width } \\
(\mathbf{m m})\end{array}$ & $\begin{array}{c}\text { Height } \\
\mathbf{( m m})\end{array}$ & $\begin{array}{c}\text { Width/ } \\
\text { Height }\end{array}$ & $\begin{array}{c}\text { Circumference } \\
\mathbf{( m m})^{*}\end{array}$ & $\begin{array}{c}\text { Effective } \\
\text { Diameter } \\
(\mathbf{m m})^{\dagger}\end{array}$ \\
\hline 1 & 8 & $1.0-1.5$ & $5.3-8.0$ & $18-19$ & $5.7-6.0$ \\
2 & 8 & $1.0-1.5$ & $5.3-8.0$ & $18-19$ & $5.7-6.0$ \\
3 & 12 & $1.0-1.5$ & $8.0-12.0$ & $26-27$ & $8.3-8.6$ \\
4 & 10 & $1.0-1.5$ & $6.7-10.0$ & $22-23$ & $7.0-7.3$ \\
5 & 8 & $1.0-1.5$ & $5.3-8.0$ & $18-19$ & $5.7-6.0$ \\
6 & 8 & 1.5 & 5.3 & $16-19$ & $5.1-6.0$ \\
\hline Mean \pm SD & $9 \pm 2$ & $1.3 \pm 0.3$ & $7.5 \pm 2.2$ & $20 \pm 3$ & $6.5 \pm 1.1$ \\
\hline
\end{tabular}

${ }^{*}$ Circumference $=2 \times$ (Width + Height $)$.

${ }^{\dagger}$ Derived assuming circular cross sections.

$\mathrm{SD}=$ standard deviation

Although the fascicular anatomy varied between nerves, the fascicular pattern of the primary targets for standing function (vasti nerves) remained consistent between specimens. Figure 2 compares the fascicular arrangement of the femoral nerve just proximal to branching between all four femoral nerves. We found fascicles from distal nerves innervating vastus medialis, vastus intermedius, and vastus lateralis consistently located on the central, and usually dorsal, portion of the proximal femoral nerve. We always found fascicles from the sensory nerves (saphenous and medial cutaneous) and rectus femoris (lateral) located on the periphery. Although we found fascicles from the sartorius nerve usually left ventrally, they were located in either the lateral, medial, or central portion of the femoral nerve. We found fascicles from the pectineus nerve usually ventrally located but mixed with fascicles from vastus medialis and vastus lateralis nerves in specimen 2 (left side).
The fascicular pattern or spatial relationship between fascicles remained consistent throughout each compound femoral nerve between the first branching point and the inguinal ligament. Figure 3 shows serial sections of a representative femoral nerve at this location. Any point on the compound femoral nerve between the first branching point and the inguinal ligament allows access to fascicles from each distal nerve and is a potential site for nerve cuff electrode placement.

The femoral nerve did not demonstrate a significant amount of fascicular joining from the distal nerve branches to the proximal compound femoral nerve. We did not observe interfascicular plexus formations. Table 5 shows the number of fascicles in the femoral nerve and the first branching point for each distal branch. Although the number of fascicles decreased proximally, we found no statistical difference between the number of fascicles in the femoral nerve and the sum of the number of branches in the distal branches. Figure 4 shows the distribution of individual fascicular diameters from the four fully mapped compound femoral nerves.

\section{DISCUSSION}

\section{Anatomy and Morphology}

The anatomy and morphology we observed remained consistent with the established literature, suggesting that our chosen specimens did not differ from the standard population and that we may consider the facscicular results representative of the general population.

We always found the major branches involved in standing (vastus medialis, vastus intermedius, and vastus lateralis nerves) centrally located, while we always found the sensory branches and the branches not implicated in standing (saphenous, medial cutaneous, sartorius, and rectus femoris nerves) located in the medial or lateral part of the branching pattern. Furthermore, branches not beneficial for standing often branched off the femoral nerve first (Table 1).

The femoral nerve branching patterns we observed in this study generally remained consistent with the literature with a few exceptions. Aizawa reported that the medial cutaneous nerve branches and vastus medialis, vastus intermedius, vastus lateralis, and rectus femoris nerves were arranged medially to laterally, respectively [24]. We also observed this pattern except in two specimens where the sartorius nerve was located between two cutaneous 


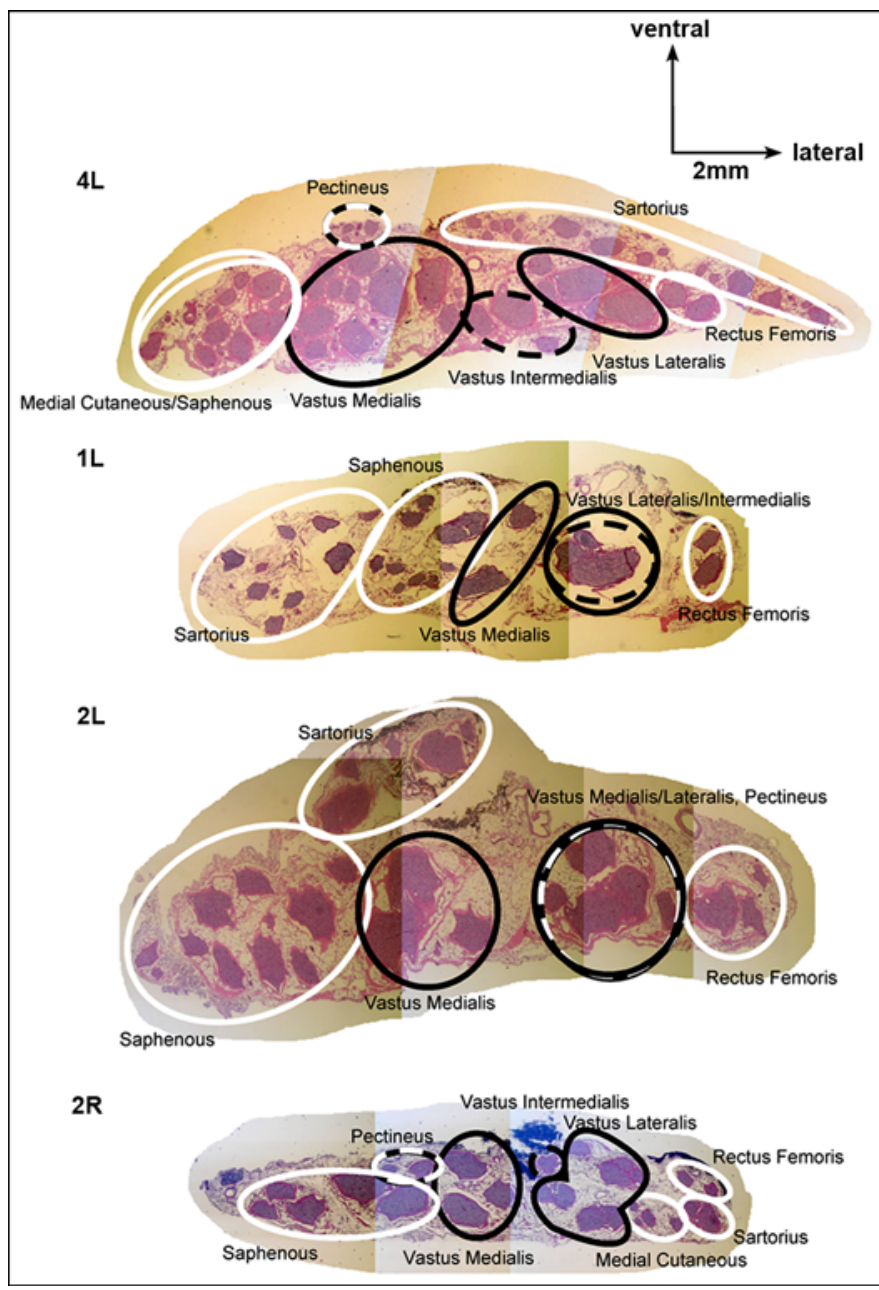

Figure 2.

Femoral nerve fascicle map comparison. We compared the fascicular patterns of the section proximal to branching in four femoral nerves (specimens 4L, 1L, 2L, and 2R). Black circles denote fascicular groups representing nerves to muscles used for standing. All are centrally located in each nerve. White circles denote fascicular groups representing nerves not beneficial for standing. All were located on periphery of nerve. Nerve cross sections are shown ventral side up. $\mathrm{L}=$ left side, $\mathrm{R}=$ right side.

branches. Aizawa found that the nerve branch to the pectineus nerve was the most medial branch of the femoral nerve in the thigh [24]. Our data concur with this; when the branch to the pectineus nerve was present, it was the most medial branch off the femoral nerve in all but one specimen, where it was the second most medial branch to leave the compound femoral nerve after the sartorius nerve. However, Aizawa reported that the branch to the pectineus nerve was the most superficial or anterior portion of the femoral nerve. While we found the branch to the pectineus nerve to

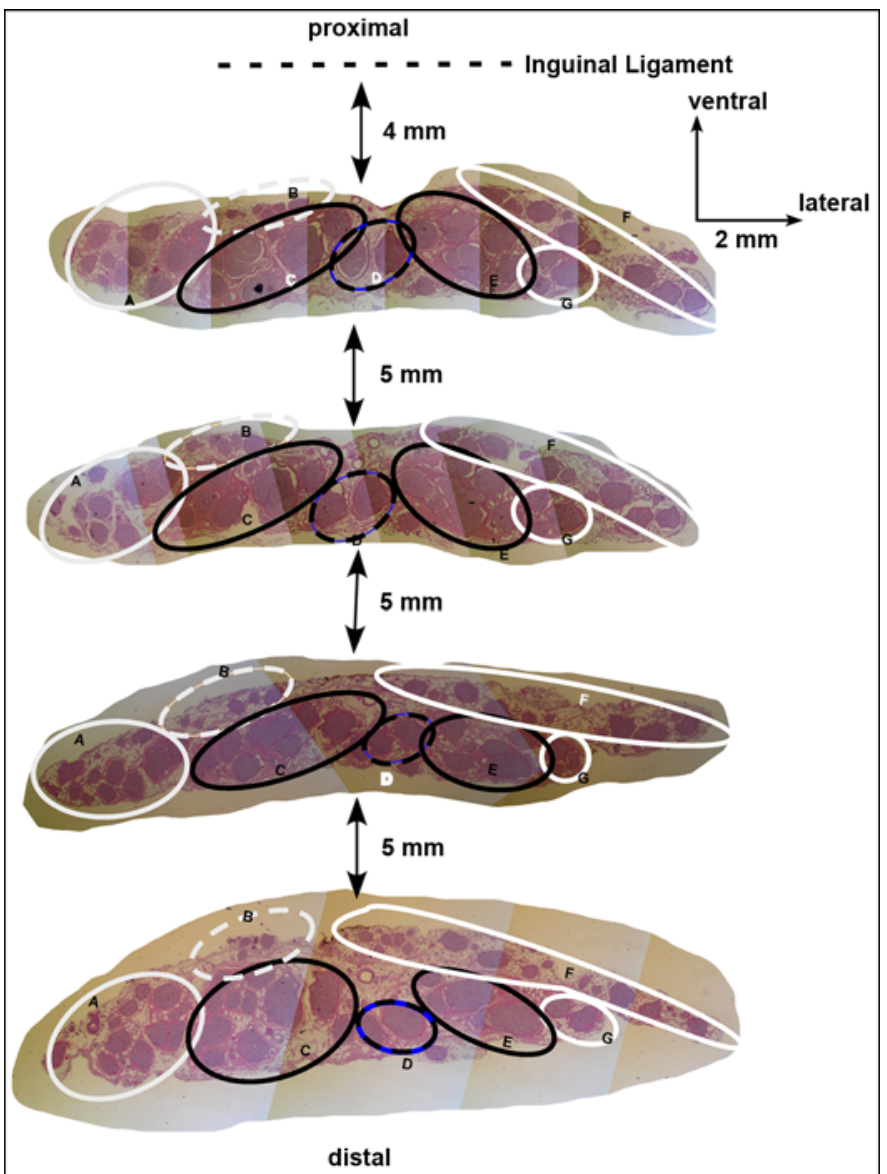

Figure 3.

Fascicle maps along compound femoral nerve (specimen 4 [left side]). We traced fascicles of compound femoral nerve proximally from point just proximal to branching to inguinal ligament. Nerve cross sections are shown ventral side up. Black circles denote fascicular groups representing nerves to muscles used for standing, while white circles represent those not used for standing. Fascicular groups represent the nerves of $\mathrm{A}=$ medial cutaneous/saphenous, $\mathrm{B}=$ pectineus, $\mathrm{C}=$ vastus medialis, $\mathrm{D}=$ vastus intermedius, $\mathrm{E}=$ vastus lateralis, $\mathrm{F}=$ sartorius, and $\mathrm{G}=$ rectus femoris.

generally be located on the superficial or anterior portion of the nerve, the branch to the sartorius nerve was always more superficial than the branch to the pectineus nerve.

Aizawa reported that the medial cutaneous nerve branch and the adductor longus branches were the first two branches to leave the femoral nerve in the thigh [24]. However, in our specimens the branch to the medial cutaneous nerve was never more proximal than the second branch, and usually occurred after the branches to the pectineus and sartorius nerves. None of our specimens had branches to adductor longus originating from the 
femoral nerve. The majority of the literature states that the adductor longus is innervated by the obturator nerve [1]. In addition, we did not identify the pectineus nerves in three specimens or the medial cutaneous nerves in two specimens. The pectineus muscle may have been innervated by the obturator nerve [1].

\section{Fascicular Anatomy}

Distal branches of the femoral nerve are represented as individual fascicles or colocalized groups of fascicles in the compound femoral nerve. The fascicular representation remained consistent with the branching pattern. We found fascicles leading to a distal nerve on the same side of the nerve that the nerve branched from. For example, we found fascicles leading to a medially branching nerve on the medial side of the nerve throughout the entire portion of the nerve from the inguinal ligament to the distal branches (Figure 1). The fascicular structure of the femoral nerve generally remained consistent between specimens, especially the vasti nerves. Always centrally and usually dorsally, we found the fascicles of the branches leading to muscles implicated in standing (vastus medialis, vastus intermedius, vastus lateralis) located on the femoral nerve (Figure 2), while we always found fascicles to the sensory branches (saphenous, medial cutaneous) and to branches innervating muscles with actions undesirable to standing (sartorius, rectus femoris) located on the periphery of the nerve. These fascicular patterns, where fascicles desirable for standing are spatially segregated from undesirable fascicles, are conducive to techniques that selectively interface with the femoral nerve.

Although early studies reported difficulties tracing fascicular anatomy along nerves [25], more recent work has demonstrated that fascicular tracing is possible [26]. Sunderland extensively studied the fascicular anatomy of the radial, medial, and ulnar nerves of the forearm and concluded that none of these nerves have a characteristic fascicular pattern because of the repeated divisions, mergers, and migration of fiber bundles [25]. However, he did report that at distal levels, fibers arranged into separate bundles according to branches. While Jabaley et al.'s studies of the same three nerves confirmed that fascicular plexus formation and interchange occur in the forearm, these interconnections do not preclude operative procedures [26]. Some nerve portions may proceed for considerable distances with no major change in position or composition; functional units and often discrete branches may remain localized in the same quadrants of nerve trunks for considerable distances and therefore may be surgically isolated [26]. We traced the fascicular pattern of the entire femoral nerve from the distal branches to the inguinal ligament using $5 \mathrm{~mm}$ sections.

While we are aware of no studies of the fascicular anatomy of the femoral nerve, Aizawa stated that the flow of nerve bundles of the femoral nerve (observed after removing the epineurium) was relatively smooth from the confluent part of the nerve to the branching part of the nerve [24]. We found that the fascicular pattern remained consistent throughout the longest section of the femoral nerve that we examined (Figure 3); therefore, the longest section of the femoral nerve with a constant pattern is a minimum of $15 \mathrm{~mm}$. Sunderland reported the longest section of the radial, medial, and ulnar nerves of the forearm with a constant pattern to be $15 \mathrm{~mm}$ [25]. Typically, the longest section with a constant pattern was 0.25 to $5.00 \mathrm{~mm}$, though individual bundles and bundle groups pursued longer courses [25].

Although we observed a reduction in the number of fascicles between the terminal branches of the femoral nerve and the femoral nerve in the thigh (Table 5), we did not find this difference statistically significant. This

Table 5.

Number of fascicles in distal nerves and section proximal to branching of femoral nerve in cadavers.

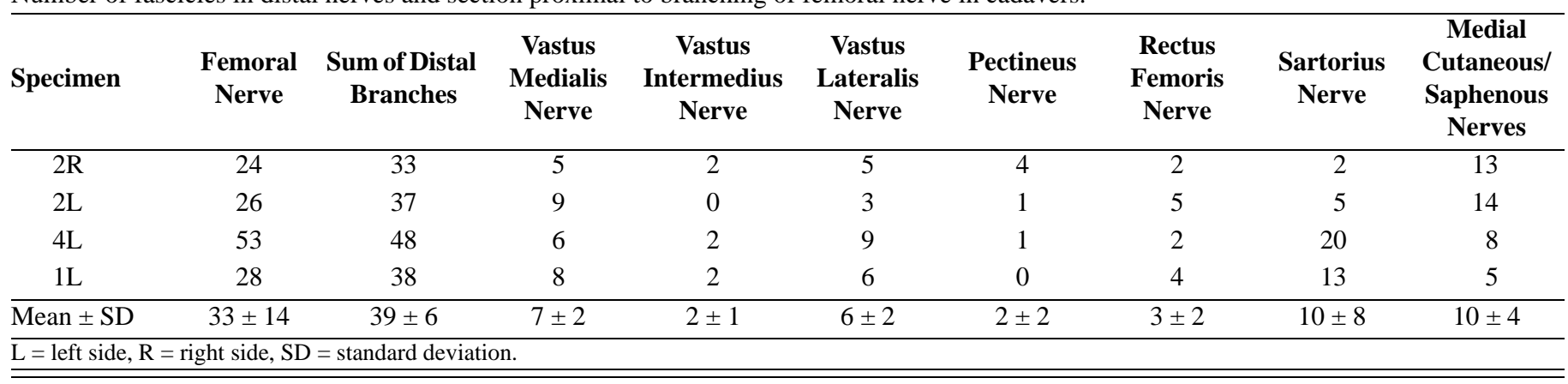




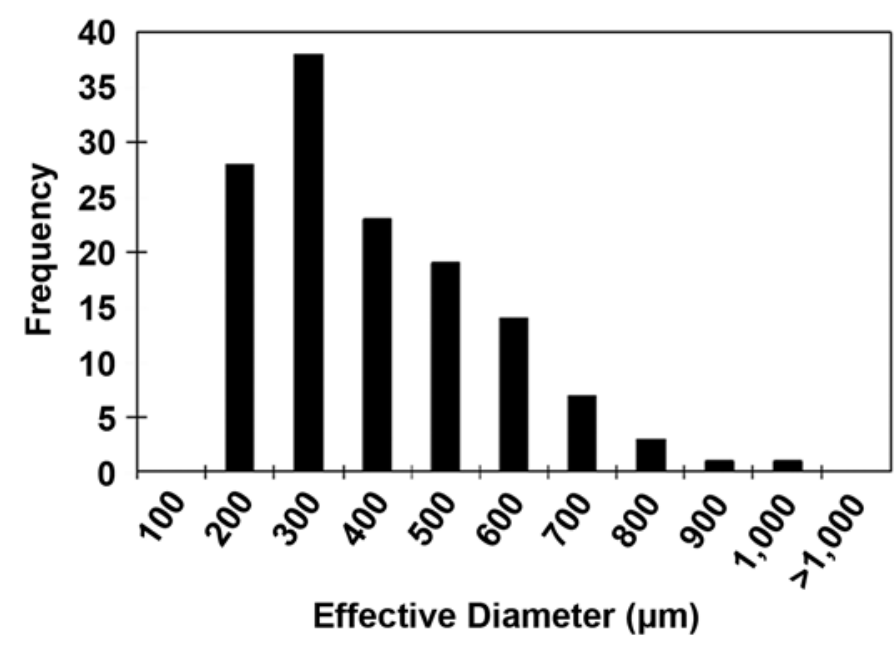

Figure 4.

Distribution of effective diameters of individual fascicles in compound femoral nerve just proximal to first branching point. Shows frequency (number of fascicles of each side) for four fully mapped specimens. Majority of fascicles (91\%) had effective diameters below $700 \mu \mathrm{m}$.

relatively small difference suggests that the amount of fascicular combining or intrafascicular plexus formation in the femoral nerve is limited at the level we examined. Although Sunderland's early work in the upper limbs reported the existence of interfascicular plexus formation [25], other studies have demonstrated interfascicular plexus formations may not be as common in the forearm [26] and may not be observed in other peripheral nerves [27]. The average number of fascicles in "the upper part" of the femoral nerve has been reported to be 36 fascicles [28]. Our data showing $33 \pm 14$ fascicles in the compound femoral nerve just proximal to branching (Table 5) are consistent with this value. The number of fascicles can vary between individuals and sides of the body, and fascicles are more numerous distal than proximal [25].

\section{Clinical Implications}

The distinct, consistent fascicular structure and crosssectional geometry of the compound femoral nerve suggest that access to distal nerves at this location is feasible. This creates opportunities to access otherwise surgically challenging nerves and stimulate multiple distal nerves with a single nerve cuff electrode able to selectively stimulate individual fascicles. Nerve morphology and fascicular anatomy data are of value for neurosurgical procedures, such as nerve anastomosis procedures for restoration of function after traumatic injury, similar to the hypoglossalfacial nerve anastomosis that is used to treat peripheral facial nerve palsy [29]. The consistent location of the compound femoral nerve at the midpoint between the ASIS and pubic symphysis allows for precise and repeatable surgical access based on readily defined surface anatomical landmarks.

The anatomy and fascicular structure of the human femoral nerve are conducive to selective stimulation with a multicontact nerve cuff electrode. The nerve diameters and branch-free lengths for the compound femoral nerve and branches (Table 2) are mostly $>2.0 \mathrm{~mm}$ in diameter and $>1.5 \mathrm{~cm}$ in branch-free length, which are suitable for effectively applying nerve cuff electrodes. Since fascicles representing muscles beneficial to standing are consistently located in the central dorsal part of the compound femoral nerve, nerve cuff electrodes that selectively stimulate these fascicles may recruit a maximal number of muscle fibers leading to leg extension without thigh or leg flexion from other femoral nerve-innervated muscles inconducive to standing.

We may obtain functional control by stimulation of distal nerve branches that innervate muscles of interest. For example, we could place nonselective electrodes on those nerve-innervating muscles providing knee extension without contraindicated muscles. We may use the presented morphology data to evaluate potential sites and the nerve cuff electrode sizes required. This approach requires additional dissection since the branching patterns can not be precisely determined a priori and few potential sites exist for functional restoration. We have used the data presented here to implant spiral nerve cuff electrodes on the femoral nerve branches in two subjects with SCI to restore standing [30].

We may also obtain functional control using intraneural dissections at the level of the femoral nerve and intraoperative stimulation to identify target fascicles and undesirable fascicles for stimulation. This approach has been used for spinal root stimulation but, to our knowledge, has not been applied in the periphery. This article provides support for intraneural dissections. The consistency of the fascicular anatomy, the functional organization, and the lack of intrafascicular plexus formation all suggest that if the fascicles could be separated safely, intraneural dissection could be feasible. However, this approach may be surgically challenging, require additional intraoperative time, and result in neural trauma. Furthermore, nerve cuff electrodes able to selectively stimulate individual fascicles within a compound nerve 
do not require intraneural dissection or a priori knowledge of the fascicular anatomy.

The relatively flat cross-section dimensions (almost 4.8:1.0 width-to-height ratio in female cadavers [Table 3]; and 7.5:1.0 in in vivo male subjects [Table 4]) of the compound femoral nerve have not been previously reported. Although we generally assumed peripheral nerves to have a circular cross section, they are commonly observed intraoperatively as flat, and relatively flat cross-sectional geometries have also been observed for the human pudendal and sciatic nerves (unpublished data from our laboratory) [27]. We could not verify in vivo nerve height measurements with a ruler or surgical pictures; therefore, we may have underestimated the actual height, especially if the center of the nerve is thicker than the ends. Formalin fixation and histological processing may have altered the crosssectional dimensions. However, the width/height ratios we observed in in vivo male subjects were similar to those we observed in female cadavers. Therefore, the relatively flat geometry is consistent.

This relatively flat geometry has a considerable effect on the design of neural prostheses and the ability to interface with the nerve surgically. Rectangular or elliptical nerve cuff electrode designs that promote a flatter nerve cross section may be more suited than traditional cylindrical nerve cuff electrodes for selective femoral nerve stimulation [19,31-33]. These designs may preserve the ordered fascicular structure and facilitate the selective activation of functionally and anatomically distinct fascicles within the compound nerve [17-19]. Indeed, modeling simulations using the fascicular anatomy presented here suggest that a multicontact flat interfacing nerve cuff electrode can selectively activate each individual fascicular group (i.e., muscle group) without activating any other muscle above threshold [23].

Although we examined a relatively small number of cadavers, the anatomy and morphology data remain consistent with earlier reports and the fascicular data remain consistent between both cadaver specimens and in vivo measurements. The intercadaver variability was relatively low and did not affect our conclusions. In addition, the presented results in the femoral nerve stayed consistent with and similar to those observed in a similar laborintensive study of the human pudendal nerve where distal nerves are represented as groups of individual fascicles in the compound pudendal nerve and a consistent fascicular orientation was observed between individuals [27].
Therefore, the results of our study are likely representative of the general population.

We expected the in vivo nerve dimensions to be larger than those histologically measured because of the shrinkage that can be anticipated with tissue fixation and processing. We could also expect nerve dimensions to be larger in male subjects, who constituted the individuals undergoing surgical exposure of their femoral nerves, while the cadaveric specimens were female. The processed histological samples in this study, however, were equivalent or larger in size than the nerves measured in vivo. Although we did not find the in vivo dimensions to be statistically lower than the postmortem values, their relative magnitude was contrary to expectations. These results suggest that tissue processing may maintain or actually increase the nerve dimensions derived from histological samples. Quantification of the effects of tissue processing will improve the prediction of nerve cuff electrode dimensions for intraoperative studies and human implants. Alternatively, it is possible that the in vivo data we collected were artificially low because of the peripheral vascular disease that necessitated surgical exposure of the femoral artery to provide access to the femoral nerve. Future studies should consider other techniques, such as quantitative imaging studies, to ascertain in vivo nerve dimensions in nondisabled volunteers age- and sex-matched to the cadaveric specimens.

\section{CONCLUSIONS}

In conclusion, this study expands our knowledge of femoral nerve morphology and clearly demonstrates the first evidence that distal nerves are represented as individual fascicles in the compound femoral nerve. The fascicular representation of distal nerves remained consistent with the branching pattern within the compound femoral nerve and reflects the function of the individual distal branches. These data allow for the evaluation of potential surgical access points, locations for nerve anastamoses or stimulating nerve cuff electrodes, and initial evaluation of the feasibility of accessing distal branches at the level of the compound femoral nerve. The femoral nerve anatomy is conducive to selective stimulation with multicontact nerve cuff electrodes. We may also use selective stimulation of the compound femoral nerve to control distal muscles and functions, such as leg extension at the knee joint. The potentially increased selectivity could improve performance of 
muscle-based electrode designs and provide more complete muscle recruitment. These improvements would be of substantial clinical benefit. Therefore, the results of this study support further development and testing of nerve cuff electrode-based interventions to restore standing function to individuals paralyzed by SCI.

\section{ACKNOWLEDGMENTS}

\section{Author Contributions:}

Study design and supervision: K. J. Gustafson, R. J. Triolo, J. A. Davis. In vivo studies: G. C. J. Pinault, J. Jean-Claude.

Cadaver studies: J. J. Neville, I. Syed.

Financial Disclosures: The authors have declared that no competing interests exist.

Funding/Support: This material was based on work supported by the Ohio Board of Regents Presidential Research Initiative, the National Institutes of Health (grants HD40298, NS40571, and EB001889), and the Department of Veterans Affairs Rehabilitation Research and Development Service (grant B3675R).

Additional Contributions: We would like to thank Matt Schiefer and Katie Polasek for their assistance. Dr. Syed is now with the Department of Orthopaedic Surgery, University of Pittsburgh Medical Center, Pittsburgh, Pennsylvania. Dr. Davis is now with the Tulane University School of Medicine, New Orleans, Louisiana.

Participant Follow-Up: The authors do not plan to inform participants of the publication of this study.

\section{REFERENCES}

1. Moore KL, Dalley AF. Clinically oriented anatomy. 4th ed. Baltimore (MD): Lippincott Williams \& Wilkins; 1999.

2. Davis JA Jr, Triolo RJ, Uhlir JP, Bhadra N, Lissy DA, Nandurkar S, Marsolais EB. Surgical technique for installing an eight-channel neuroprosthesis for standing. Clin Orthop Relat Res. 2001;(385):237-52. [PMID: 11302320] DOI:10.1097/00003086-200104000-00035

3. Kobetic R, Triolo RJ, Marsolais EB. Muscle selection and walking performance of multichannel FES systems for ambulation in paraplegia. IEEE Trans Rehabil Eng. 1997; 5(1):23-29. [PMID: 9086382]

DOI:10.1109/86.559346

4. Uhlir JP, Triolo RJ, Kobetic R. The use of selective electrical stimulation of the quadriceps to improve standing function in paraplegia. IEEE Trans Rehabil Eng. 2000;8(4): 514-22. [PMID: 11204043]

DOI:10.1109/86.895955

5. Kobetic R, Triolo RJ, Uhlir JP, Bieri C, Wibowo M, Polando G, Marsolais EB, Davis JA Jr, Ferguson KA. Implanted functional electrical stimulation system for mobility in paraplegia: A follow-up case report. IEEE Trans Rehabil Eng.
1999;7(4):390-98. [PMID: 10609626]

DOI:10.1109/86.808942

6. Sharma M, Marsolais EB, Polando G, Triolo RJ, Davis JA Jr, Bhadra N, Uhlir JP. Implantation of a 16-channel functional electrical stimulation walking system. Clin Orthop Relat Res. 1998;(347):236-42. [PMID: 9520896] DOI:10.1097/00003086-199802000-00029

7. Triolo R, Wibowo M, Uhlir J, Kobetic R, Kirsch R. Effects of stimulated hip extension moment and position on upperlimb support forces during FNS-induced standing-A technical note. J Rehabil Res Dev. 2001;38(5):545-55. [PMID: 11732832]

8. Akers JM, Peckham PH, Keith MW, Merritt K. Tissue response to chronically stimulated implanted epimysial and intramuscular electrodes. IEEE Trans Rehabil Eng. 1997; 5(2):207-20. [PMID: 9184906] DOI:10.1109/86.593301

9. Grandjean PA, Mortimer JT. Recruitment properties of monopolar and bipolar epimysial electrodes. Ann Biomed Eng. 1986;14(1):53-66. [PMID: 3706855]

DOI:10.1007/BF02364648

10. Memberg WD, Peckham PH, Keith MW. A surgicallyimplanted intramuscular electrode for an implantable neuromuscular stimulation system. IEEE Trans Rehabil Eng. 1994; 2(2):80-91. DOI:10.1109/86.313149

11. Kilgore KL, Peckham PH, Keith MW, Thrope GB, Wuolle KS, Bryden AM, Hart RL. An implanted upper-extremity neuroprosthesis. Follow-up of five patients. J Bone Joint Surg Am. 1997;79(4):533-41. [PMID: 9111397]

12. Peckham PH, Keith MW, Kilgore KL, Grill JH, Wuolle KS, Thrope GB, Gorman P, Hobby J, Mulcahey MJ, Carroll S, Hentz VR, Wiegner A; Implantable Neuroprosthesis Research Group. Efficacy of an implanted neuroprosthesis for restoring hand grasp in tetraplegia: A multicenter study. Arch Phys Med Rehabil. 2001;82(10):1380-88. [PMID: 11588741] DOI:10.1053/apmr.2001.25910

13. Davis JA Jr, Triolo RJ, Uhlir J, Bieri C, Rohde L, Lissy D, Kukke S. Preliminary performance of a surgically implanted neuroprosthesis for standing and transfers-Where do we stand? J Rehabil Res Dev. 2001;38(6):609-17.

[PMID: 11767968]

14. Marsolais EB, Scheiner A, Miller PC, Kobetic R, Daly JJ. Augmentation of transfers for a quadriplegic patient using an implanted FNS system. Case report. Paraplegia. 1994; 32(8):573-79. [PMID: 7970864]

15. Triolo RJ, Bieri C, Uhlir J, Kobetic R, Scheiner A, Marsolais EB. Implanted Functional Neuromuscular Stimulation systems for individuals with cervical spinal cord injuries: Clinical case reports. Arch Phys Med Rehabil. 1996;77(11): 1119-28. [PMID: 8931521] DOI:10.1016/S0003-9993(96)90133-1 
16. Kagaya H, Shimada Y, Ebata K, Sato M, Sato K, Yukawa T, Obinata G. Restoration and analysis of standing-up in complete paraplegia utilizing functional electrical stimulation. Arch Phys Med Rehabil. 1995;76(9):876-81. [PMID: 7668962] DOI:10.1016/S0003-9993(95)80556-7

17. Choi AQ, Cavanaugh JK, Durand DM. Selectivity of multiple-contact nerve cuff electrodes: A simulation analysis. IEEE Trans Biomed Eng. 2001;48(2):165-72. [PMID: 11296872] DOI:10.1109/10.909637

18. Grill WM Jr, Mortimer JT. Quantification of recruitment properties of multiple contact cuff electrodes. IEEE Trans Rehabil Eng. 1996;4(2):49-62. [PMID: 8798072] DOI:10.1109/86.506402

19. Tyler DJ, Durand DM. Functionally selective peripheral nerve stimulation with a flat interface nerve electrode. IEEE Trans Neural Syst Rehabil Eng. 2002;10(4):294-303. [PMID: 12611367] DOI:10.1109/TNSRE.2002.806840

20. Grinberg Y, Schiefer MA, Tyler DJ, Gustafson KJ. Fascicular perineurium thickness, size, and position affect model predictions of neural excitation. IEEE Trans Neural Syst Rehabil Eng. 2008;16(6):572-81. [PMID: 19144589] DOI:10.1109/TNSRE.2008.2010348

21. Grill WM, Mortimer JT. Neural and connective tissue response to long-term implantation of multiple contact nerve cuff electrodes. J Biomed Mater Res. 2000;50(2):215-26. [PMID: 10679687] DOI:10.1002/(SICI)1097-4636(200005)50:2<215::AIDJBM17>3.0.CO;2-A

22. Naples GG, Mortimer JT, Yuen TG. Overview of peripheral nerve electrode design and implantation. In: Agnew WF, McCreery DB, editors. Neural prostheses: Fundamental studies. Englewood Cliffs (NJ): Prentice Hall; 1990. p. 107-44.

23. Schiefer MA, Triolo RJ, Tyler DJ. A model of selective activation of the femoral nerve with a flat interface nerve electrode for a lower extremity neuroprosthesis. IEEE Trans Neural Syst Rehabil Eng. 2008;16(2):195-204. [PMID: 18403289] DOI:10.1109/TNSRE.2008.918425

24. Aizawa Y. On the organization of the Plexus lumbalis. I. On the recognition of the three-layered divisions and the systematic description of the branches of the human femo- ral nerve. Okajimas Folia Anat Jpn. 1992;69(1):35-74. [PMID: 1620529]

25. Sunderland S. The intraneural topography of the radial, median, and ulnar nerves. Brain. 1945;68(4):243-98.

DOI:10.1093/brain/68.4.243

26. Jabaley ME, Wallace WH, Heckler FR. Internal topography of major nerves of the forearm and hand: A current view. J Hand Surg Am. 1980;5(1):1-18. [PMID: 7365209]

27. Gustafson KJ, Zelkovic PF, Feng AH, Draper CE, Bodner DR, Grill WM. Fascicular anatomy and surgical access of the human pudendal nerve. World J Urol. 2005;23(6):411-18.

[PMID: 16333625] DOI:10.1007/s00345-005-0032-4

28. Urbanowicz Z. Femoral nerve fascicles in the human postfetal life. Folia Morphol (Warsz). 1981;39(3):283-91. [PMID: 6973517]

29. Salame K, Ouaknine GE, Arensburg B, Rochkind S. Microsurgical anatomy of the facial nerve trunk. Clin Anat. 2002;15(2):93-99. [PMID: 11877786] DOI:10.1002/ca.1102

30. Fisher LE, Miller ME, Bailey SN, Davis JA Jr, Anderson JS, Rhode L, Tyler DJ, Triolo RJ. Standing after spinal cord injury with four-contact nerve-cuff electrodes for quadriceps stimulation. IEEE Trans Neural Syst Rehabil Eng. 2008;16(5):473-78. [PMID: 18990650]

DOI:10.1109/TNSRE.2008.2003390

31. Leventhal DK, Durand DM. Subfascicle stimulation selectivity with the flat interface nerve electrode. Ann Biomed Eng. 2003;31(6):643-52. [PMID: 12797613]

DOI:10.1114/1.1569266

32. Tarler MD, Mortimer JT. Selective and independent activation of four motor fascicles using a four contact nerve-cuff electrode. IEEE Trans Neural Syst Rehabil Eng. 2004; 12(2):251-57. [PMID: 15218938] DOI:10.1109/TNSRE.2004.828415

33. Tyler DJ, Durand DM. Chronic response of the rat sciatic nerve to the flat interface nerve electrode. Ann Biomed Eng. 2003;31(6):633-42. [PMID: 12797612] DOI:10.1114/1.1569263

Submitted for publication August 8, 2008. Accepted in revised form April 23, 2009. 